\begin{tabular}{|c|c|}
\hline Title & Geometric Interpretation of Lagrangian Equivalence \\
\hline Author(s) & Izumiya, Shyuichi \\
\hline Citation & $\begin{array}{l}\text { Canadian mathematical bulletin, 59(4), 806-812 } \\
\text { https://doi.org/10.4153/CMB-2016-056-2 }\end{array}$ \\
\hline Issue Date & 2016-12 \\
\hline Doc URL & http:/hdl.handle.net/2115/64074 \\
\hline Rights & $\begin{array}{l}\text { First published in Canadian Mathematical Bulletin at http://dx.doi.org/10.4153/CMB-2016-056-2. Copyright @ 2016, } \\
\text { Canadian Mathematical Society }\end{array}$ \\
\hline Type & article \\
\hline File Information & Lagequivrev.pdf \\
\hline
\end{tabular}

Instructions for use 


\title{
Geometric interpretation of Lagrangian equivalence
}

\author{
Shyuichi IZUMIYA
}

August 13, 2016

\begin{abstract}
As an application of the theory of graph-like Legendrian unfoldings, relations of the hidden structures of caustics and wave front propagations are revealed.
\end{abstract}

\section{Introduction}

Lagrangian equivalence among Lagrangian submanifold germs in the cotangent bundle was introduced for the study of oscillatory integrals on caustics (cf., [2, 3, 4]). It has been known that caustic equivalence (i.e., diffeomorphic caustics) does not imply Lagrangian equivalence. This is one of the main differences from the theory of Legendrian singularities. In the theory of Legendrian singularities, wave front equivalence (i.e., diffeomorphic wave fronts) implies Legendrian equivalence generically [11]. Therefore, Legendrian equivalence is geometric equivalence in this sense. In the real world, the caustics given by reflected rays are visible. However, the wave front propagations are not visible. Therefore, we can say that there are hidden structures behind the picture of caustics (cf., [10]). In fact, caustics are a subject of classical physics (i.e., geometric optics). However, the corresponding Lagrangian submanifold is deeply related to the semi-classical approximation of quantum mechanics.

On the other hand, the notion of graph-like Legendrian unfoldings was introduced in [5]. It belongs to a special class of big Legendrian submanifolds (wave front propagations) which Zakalyukin introduced in [11]. There have been some developments on this theory during past two decades $[5,6,7,9]$. There appeared several equivalence relations among big Legendrian submanifolds in these articles for different purposes. However, the relation to Lagrangian equivalence has not been clear so far. One of the main results in this paper are Theorems 4.3 and 4.5 which reveal the relation between caustics and wave front propagations. For the proof of Theorem 4.3, we show Propositions 4.1 and 4.2. Actually, Proposition 4.1 has been proved in [7] by using the notion of generating families which is a strong tool for the study of Lagrangian equivalence. Proposition 4.2 is the key assertion in this paper. We avoid to use the notion of generating families to shorten the paper.

2010 Mathematics Subject classification. Primary 58K05,57R45 ; Secondary 58K60

Keywords. wave front propagations, big wave fronts, graph-like Legendrian unfoldings, Caustics This work was supported by JSPS KAKENHI Grant Number26287009. 


\section{$2 \quad$ Lagrangian singularities}

We briefly describe the basic notions of the local theory of Lagrangian singularities due to [1]. We consider the cotangent bundle $\pi: T^{*} \mathbb{R}^{n} \rightarrow \mathbb{R}^{n}$ over $\mathbb{R}^{n}$. Let $(x, p)=$ $\left(x_{1}, \ldots, x_{n}, p_{1}, \ldots, p_{n}\right)$ be the canonical coordinates on $T^{*} \mathbb{R}^{n}$. Then the canonical symplectic structure on $T^{*} \mathbb{R}^{n}$ is given by the canonical two form $\omega=\sum_{i=1}^{n} d p_{i} \wedge d x_{i}$. Let $i: L \subset T^{*} \mathbb{R}^{n}$ be a submanifold. We say that $i$ is a Lagrangian submanifold if $\operatorname{dim} L=n$ and $i^{*} \omega=0$. In this case, the set of critical values of $\pi \circ i$ is called the caustic of $i: L \subset T^{*} \mathbb{R}^{n}$, which is denoted by $C_{L}$.

We now define a natural equivalence relation among Lagrangian submanifold germs. Let $i:(L, p) \subset\left(T^{*} \mathbb{R}^{n}, p\right)$ and $i^{\prime}:\left(L^{\prime}, p^{\prime}\right) \subset\left(T^{*} \mathbb{R}^{n}, p^{\prime}\right)$ be Lagrangian submanifold germs. Then we say that $i$ and $i^{\prime}$ are Lagrangian equivalent if there exist a diffeomorphism germ $\sigma:(L, p) \rightarrow\left(L^{\prime}, p^{\prime}\right)$, a symplectic diffeomorphism germ $\hat{\tau}:\left(T^{*} \mathbb{R}^{n}, p\right) \rightarrow\left(T^{*} \mathbb{R}^{n}, p^{\prime}\right)$ and a diffeomorphism germ $\tau:\left(\mathbb{R}^{n}, \pi(p)\right) \rightarrow\left(\mathbb{R}^{n}, \pi\left(p^{\prime}\right)\right)$ such that $\hat{\tau} \circ i=i^{\prime} \circ \sigma$ and $\pi \circ \hat{\tau}=\tau \circ \pi$, where $\pi:\left(T^{*} \mathbb{R}^{n}, p\right) \rightarrow\left(\mathbb{R}^{n}, \pi(p)\right)$ is the canonical projection. Here $\hat{\tau}$ is said to be a symplectic diffeomorphism germ if it is a diffeomorphism germ such that $\hat{\tau}^{*} \omega=\omega$. We also say that $i$ and $i^{\prime}$ are caustic equivalent if $C_{L}$ and $C_{L^{\prime}}$ are diffeomorphic. By definition, if $i$ and $i^{\prime}$ are Lagrangian equivalent then $i$ and $i^{\prime}$ are caustic equivalent. In general, the converse does not hold. This is the reason why we have no geometric interpretation of Lagrangian equivalence so far. There is the notion of Lagrangian stability of a Lagrangian submanifold germ (cf., [1]). Here, we do not need the exact definition, so that we omit the definition.

\section{Theory of the wave front propagations}

In this section we describe the basic notions of the theory of wave front propagations (for details, see $[1,5,11,12]$, etc). We start to consider the general theory of Legendrian singularities. Let $\bar{\pi}: P T^{*}\left(\mathbb{R}^{m}\right) \longrightarrow \mathbb{R}^{m}$ be the projective cotangent bundle over $\mathbb{R}^{m}$. This fibration can be considered as a Legendrian fibration with the canonical contact structure $K$ on $P T^{*}\left(\mathbb{R}^{m}\right)$. We have the trivialization $P T^{*}\left(\mathbb{R}^{m}\right) \cong \mathbb{R}^{m} \times P\left(\mathbb{R}^{m *}\right)$ and we call $(x,[\xi])$ homogeneous coordinates, where $x=\left(x_{1}, \ldots, x_{m}\right) \in \mathbb{R}^{m}$ and $[\xi]=\left[\xi_{1}: \cdots: \xi_{m}\right]$ are homogeneous coordinates of the dual projective space $P\left(\mathbb{R}^{m *}\right)$. Let $\Phi:\left(\mathbb{R}^{m}, 0\right) \longrightarrow$ $\left(\mathbb{R}^{m}, 0\right)$ be a diffeomorphism germ. Then we have a unique contact diffeomorphism germ $\widehat{\Phi}: P T^{*} \mathbb{R}^{m} \longrightarrow P T^{*} \mathbb{R}^{m}$ defined by $\widehat{\Phi}(x,[\xi])=\left(\Phi(x),\left[\xi \circ d_{\Phi(x)}\left(\Phi^{-1}\right)\right]\right)$. We say that $\widehat{\Phi}$ is a contact diffeomorphism if $d \widehat{\Phi}_{((x,[\xi])}\left(K_{((x,[\xi])}\right)=K_{\widehat{\Phi}(x,[\xi])}$. We call $\widehat{\Phi}$ the contact lift of $\Phi$. A submanifold $i: \mathscr{L} \subset P T^{*}\left(\mathbb{R}^{m}\right)$ is said to be a Legendrian submanifold if $\operatorname{dim} \mathscr{L}=m-1$ and $d i_{p}\left(T_{p} \mathscr{L}\right) \subset K_{i(p)}$ for any $p \in L$. We also call $\bar{\pi} \circ i=\left.\bar{\pi}\right|_{\mathscr{L}}: \mathscr{L} \longrightarrow \mathbb{R}^{m}$ a Legendrian map and $W(\mathscr{L})=\bar{\pi}(\mathscr{L})$ a wave front of $i: \mathscr{L} \subset P T^{*}\left(\mathbb{R}^{m}\right)$. We say that a point $p \in \mathscr{L}$ is a Legendrian singular point if $\operatorname{rank} d\left(\left.\bar{\pi}\right|_{\mathscr{L}}\right)_{p}<m-1$. In this case $\bar{\pi}(p)$ is the singular point of $W(\mathscr{L})$. We say that two Legendrian submanifold germs $\mathscr{L}$ and $\mathscr{L}^{\prime}$ are Legendrian equivalent if there exists a diffeomorphism germ $\Phi:\left(\mathbb{R}^{m}, 0\right) \longrightarrow\left(\mathbb{R}^{m}, 0\right)$ such that $\widehat{\Phi}(\mathscr{L})=\mathscr{L}^{\prime}$ as set germs.

We now consider the case when $m=n+1$ and distinguish space and time coordinates, so that we write $\mathbb{R}^{n+1}=\mathbb{R}^{n} \times \mathbb{R}$ and coordinates are denoted by $(x, t)=\left(x_{1}, \ldots, x_{n}, t\right) \in$ $\mathbb{R}^{n} \times \mathbb{R}$. For the projective cotangent bundle $\bar{\pi}: P T^{*}\left(\mathbb{R}^{n} \times \mathbb{R}\right) \rightarrow \mathbb{R}^{n} \times \mathbb{R}$, we have 
homogeneous coordinates $\left(\left(x_{1}, \ldots, x_{n}, t\right),\left[\xi_{1}: \cdots: \xi_{n}: \tau\right]\right)$ of $P T^{*}\left(\mathbb{R}^{n} \times \mathbb{R}\right) \cong\left(\mathbb{R}^{n} \times \mathbb{R}\right) \times$ $P\left(\left(\mathbb{R}^{n} \times \mathbb{R}\right)^{*}\right)$,

For a Legendrian submanifold $i: \mathscr{L} \subset P T^{*}\left(\mathbb{R}^{n} \times \mathbb{R}\right)$, the corresponding wave front $\bar{\pi} \circ i(\mathscr{L})=W(\mathscr{L})$ is called a big wave front. We call

$$
W_{t}(\mathscr{L})=\pi_{1}\left(\pi_{2}^{-1}(t) \cap W(\mathscr{L})\right) \quad(t \in \mathbb{R})
$$

a momentary front (or, a small front) for each $t \in \mathbb{R}$, where $\pi_{1}: \mathbb{R}^{n} \times \mathbb{R} \rightarrow \mathbb{R}^{n}$ and $\pi_{2}: \mathbb{R}^{n} \times \mathbb{R} \rightarrow \mathbb{R}$ are the canonical projections defined by $\pi_{1}(x, t)=x$ and $\pi_{2}(x, t)=t$ respectively. In this sense, we call $\mathscr{L}$ a big Legendrian submanifold. We say that a point $p \in \mathscr{L}$ is a space-singular point if rank $d\left(\left.\pi_{1} \circ \bar{\pi}\right|_{\mathscr{L}}\right)_{p}<n$ and a time-singular point if $\operatorname{rank} d\left(\pi_{2} \circ \bar{\pi} \mid \mathscr{L}\right)_{p}=0$, respectively. By definition, if $p \in \mathscr{L}$ is a Legendrian singular point, then it is a space-singular point of $\mathscr{L}$. The discriminant of the family $\left\{W_{t}(\mathscr{L})\right\}_{t \in \mathbb{R}}$ is defined as the image of singular points of $\left.\pi_{1}\right|_{W(\mathscr{L})}$. In the general case, the discriminant consists of three components: the caustic $C_{\mathscr{L}}=\pi_{1}(\Sigma(W(\mathscr{L}))$, where $\Sigma(W(\mathscr{L}))$ is the set of singular points of $W(\mathscr{L})$ (i.e, the critical value set of the Legendrian mapping $\left.\bar{\pi}\right|_{\mathscr{L}}$ ), the Maxwell stratified set $M_{\mathscr{L}}$, the projection of the closure of the self intersection set of $W(\mathscr{L})$; and also of the critical value set $\Delta_{\mathscr{L}}$ of $\left.\pi_{1}\right|_{W(\mathscr{L}) \backslash \Sigma(W(\mathscr{L}))}$.

We now consider an equivalence relation among big Legendrian submanifolds which was independently introduced in $[6,12]$ for different purposes: Let $i:\left(\mathscr{L}, p_{0}\right) \subset\left(P T^{*}\left(\mathbb{R}^{n} \times\right.\right.$ $\left.\mathbb{R}), p_{0}\right)$ and $i^{\prime}:\left(\mathscr{L}^{\prime}, p_{0}^{\prime}\right) \subset\left(P T^{*}\left(\mathbb{R}^{n} \times \mathbb{R}\right), p_{0}^{\prime}\right)$ be big Legendrian submanifold germs. Then we say that $i$ and $i^{\prime}$ are strictly parametrized ${ }^{+}$Legendrian equivalent (or, briefly $S . P^{+}$Legendrian equivalent) if there exists a diffeomorphism germs $\Phi:\left(\mathbb{R}^{n} \times \mathbb{R}, \bar{\pi}\left(p_{0}\right)\right) \rightarrow$ $\left(\mathbb{R}^{n} \times \mathbb{R}, \bar{\pi}\left(p_{0}^{\prime}\right)\right)$ of the form $\Phi(x, t)=\left(\phi_{1}(x), t+\alpha(x)\right)$ such that $\widehat{\Phi}(\mathscr{L})=\mathscr{L}^{\prime}$ as set germs, where $\widehat{\Phi}:\left(P T^{*}\left(\mathbb{R}^{n} \times \mathbb{R}\right), p_{0}\right) \rightarrow\left(P T^{*}\left(\mathbb{R}^{n} \times \mathbb{R}\right), p_{0}^{\prime}\right)$ is the unique contact lift of $\Phi$. We can also define the notion of stability of Legendrian submanifold germs with respect to the above equivalence relation which is analogous to the stability of Legendrian submanifold germs with respect to Legendrian equivalence (cf. [1, Part III]).

On the other hand, concerning the discriminant, we define the following equivalence relation among big wave front germs. Let $i:\left(\mathscr{L}, p_{0}\right) \subset\left(P T^{*}\left(\mathbb{R}^{n} \times \mathbb{R}\right), p_{0}\right)$ and $i^{\prime}$ : $\left(\mathscr{L}^{\prime}, p_{0}^{\prime}\right) \subset\left(P T^{*}\left(\mathbb{R}^{n} \times \mathbb{R}\right), p_{0}^{\prime}\right)$ be big Legendrian submanifold germs. We say that $W(\mathscr{L})$ and $W\left(\mathscr{L}^{\prime}\right)$ are $S . P^{+}$-diffeomorphic if there exists a diffeomorphism germ $\Phi:\left(\mathbb{R}^{n} \times\right.$ $\left.\mathbb{R}, \bar{\pi}\left(p_{0}\right)\right) \rightarrow\left(\mathbb{R}^{n} \times \mathbb{R}, \bar{\pi}\left(p_{0}^{\prime}\right)\right)$ of the form $\Phi(x, t)=\left(\phi_{1}(x), t+\alpha(x)\right)$ such that $\Phi(W(\mathscr{L}))=$ $W\left(\mathscr{L}^{\prime}\right)$ as set germs. We also call $\Phi$ a $S . P^{+}$-diffeomorphism germ. We remark that $S . P^{+}$-diffeomorphism among big wave front germs preserves the diffeomorphism types of $C_{\mathscr{L}} \cup M_{\mathscr{L}} \cup \Delta_{\mathscr{L}}$. Since the Legendrian submanifold germ is uniquely determined on the regular part of the wave front set, we have the following proposition as an easy corollary of the result in [11].

Proposition 3.1 Let $i:\left(\mathscr{L}, p_{0}\right) \subset\left(P T^{*}\left(\mathbb{R}^{n} \times \mathbb{R}\right), p_{0}\right)$ and $i^{\prime}:\left(\mathscr{L}^{\prime}, p_{0}^{\prime}\right) \subset\left(P T^{*}\left(\mathbb{R}^{n} \times\right.\right.$ $\left.\mathbb{R}), p_{0}^{\prime}\right)$ be big Legendrian submanifold germs such that the sets of critical points of $\bar{\pi} \circ i, \bar{\pi} \circ i^{\prime}$ are nowhere dense respectively. Then $i$ and $i^{\prime}$ are $S . P^{+}$-Legendrian equivalent if and only if $\left(W(\mathscr{L}), \bar{\pi}\left(p_{0}\right)\right)$ and $\left(W\left(\mathscr{L}^{\prime}\right), \bar{\pi}\left(p_{0}^{\prime}\right)\right)$ are $S . P^{+}$-diffeomorphic. 


\section{Graph-like Legendrian unfoldings}

In this section we explain the theory of graph-like Legendrian unfoldings and prove the main theorems. The notion of graph-like Legendrian unfoldings was introduced in [5]. A graph-like Legendrian unfolding belongs to a special class of big Legendrian submanifolds.

We remark that $P T^{*}\left(\mathbb{R}^{n} \times \mathbb{R}\right)$ is a fiber-wise compactification of the 1-jet space as follows: We consider an affine open subset $U_{\tau}=\{((x, t),[\xi: \tau]) \mid \tau \neq 0\}$ of $P T^{*}\left(\mathbb{R}^{n} \times \mathbb{R}\right)$. For any $((x, t),[\xi: \tau]) \in U_{\tau}$, we have

$$
\left(\left(x_{1}, \ldots, x_{n}, t\right),\left[\xi_{1}: \cdots: \xi_{n}: \tau\right]\right)=\left(\left(x_{1}, \ldots, x_{n}, t\right),\left[-\left(\xi_{1} / \tau\right): \cdots:-\left(\xi_{n} / \tau\right):-1\right]\right),
$$

so that we may adopt the corresponding affine coordinates $\left(\left(x_{1}, \ldots, x_{n}, t\right),\left(p_{1}, \ldots, p_{n}\right)\right)$, where $p_{i}=-\xi_{i} / \tau$. On $U_{\tau}$ we have $\theta^{-1}(0)=\left.K\right|_{U_{\tau}}$, where $\theta=d t-\sum_{i=1}^{n} p_{i} d x_{i}$. This means that $U_{\tau}$ can be identified with the 1 -jet space which is denoted by $J_{G A}^{1}\left(\mathbb{R}^{n}, \mathbb{R}\right) \subset P T^{*}\left(\mathbb{R}^{n} \times\right.$ $\mathbb{R})$. We call the above coordinates a system of graph-like affine coordinates. Throughout this paper, we use this identification. A big Legendrian submanifold $i: \mathscr{L} \subset P T^{*}\left(\mathbb{R}^{n} \times \mathbb{R}\right)$ is said to be a graph-like Legendrian unfolding if $\mathscr{L} \subset J_{G A}^{1}\left(\mathbb{R}^{n}, \mathbb{R}\right)$. We call $W(\mathscr{L})=\bar{\pi}(\mathscr{L})$ a graph-like wave front of $\mathscr{L}$, where $\bar{\pi}: J_{G A}^{1}\left(\mathbb{R}^{n}, \mathbb{R}\right) \longrightarrow \mathbb{R}^{n} \times \mathbb{R}$ is the canonical projection. We define a mapping $\Pi: J_{G A}^{1}\left(\mathbb{R}^{n}, \mathbb{R}\right) \longrightarrow T^{*} \mathbb{R}^{n}$ by $\Pi(x, t, p)=(x, p)$, where $(x, t, p)=$ $\left(x_{1}, \ldots, x_{n}, t, p_{1}, \ldots, p_{n}\right)$. Then we have the following proposition. In [7] we have shown that $\left.\Pi\right|_{\mathscr{L}}: \mathscr{L} \longrightarrow T^{*} \mathbb{R}^{n}$ is immersive, so that $\Pi(\mathscr{L})$ is a Lagrangian submanifold in $T^{*} \mathbb{R}^{n}$. Moreover, the discriminant of the family of momentary fronts is $C_{\mathscr{L}} \cup M_{\mathscr{L}}$ for a graphlike Legendrian unfolding $\mathscr{L} \subset J_{G A}^{1}\left(\mathbb{R}^{n}, \mathbb{R}\right)$. By using the notion of generating families, we can show the following assertion: For any Lagrangian submanifold germ $(L, z) \subset$ $T^{*} \mathbb{R}^{n}$, there exists a graph-like Legendrian unfolding germ $(\mathscr{L}, p) \subset J_{G A}^{1}\left(\mathbb{R}^{n}, \mathbb{R}\right)$ such that $(\Pi(\mathscr{L}), \Pi(p))=(L, z)$. We now compare the equivalence relations between graph-like Legendrian unfoldings and induced Lagrangian submanifold germs. By using the notion of graph-like generating families of graph-like Legendrian unfoldings and generating families of Lagrangian submanifold germs respectively, we showed the following proposition in [7].

Proposition 4.1 ([7]) Let $\left(\mathscr{L}_{1}, p_{1}\right),\left(\mathscr{L}_{2}, p_{2}\right)$ be graph-like Legendrian unfoldings. If $\left(\Pi\left(\mathscr{L}_{1}\right), \Pi\left(p_{1}\right)\right)$ and $\left(\Pi\left(\mathscr{L}_{2}\right) . \Pi\left(p_{2}\right)\right)$ are Lagrangian equivalent, then $\left(\mathscr{L}_{1}, p_{1}\right)$ and $\left(\mathscr{L}_{2}, p_{2}\right)$ are $S . P^{+}$-Legendrian equivalent.

The above proposition asserts that Lagrangian equivalence is a stronger equivalence relation than $S . P^{+}$-Legendrian equivalence. The $S . P^{+}$-Legendrian equivalence relation among graph-like Legendrian unfoldings preserves both the diffeomorphism types of caustics and Maxwell stratified sets. On the other hand, if we observe the real caustics of rays, we cannot observe the structure of wave front propagations and the Maxwell stratified sets. In this sense, there are hidden structures behind the picture of real caustics. By the above proposition, Lagrangian equivalence preserves not only the diffeomorphism type of caustics, but also the hidden geometric structure of wave front propagations. It has been believed that the converse assertion of Proposition 4.1 does not hold since a long time ago at least for me. Actually, [8] showed the converse assertion only for the case that $\Pi\left(\mathscr{L}_{1}\right)$ and $\Pi\left(\mathscr{L}_{2}\right)$ are Lagrange stable. Therefore, we have not tried to show the converse assertion so far. However, we have the following result. 
Proposition 4.2 Let $\left(\mathscr{L}_{1}, p_{1}\right),\left(\mathscr{L}_{2}, p_{2}\right)$ be graph-like Legendrian unfoldings. If $\left(\mathscr{L}_{1}, p_{1}\right)$ and $\left(\mathscr{L}_{2}, p_{2}\right)$ are $S . P^{+}{ }_{-}$Legendrian equivalent, then $\left(\Pi\left(\mathscr{L}_{1}\right), \Pi\left(p_{1}\right)\right)$ and $\left(\Pi\left(\mathscr{L}_{2}\right), \Pi\left(p_{2}\right)\right)$ are Lagrangian equivalent.

Proof. In order to simplify the arguments, we use $x=\left(x_{1}, \ldots, x_{n}\right), \xi=\left(\xi_{1}, \ldots, \xi_{n}\right)$ and $p=\left(p_{1}, \ldots, p_{n}\right)$. With this notation, we write that $\xi \cdot x=\sum_{i=1}^{n} \xi_{i} x_{i}$ and $\theta=d t-p \cdot d x=$ $d t-\sum_{i=1}^{n} p_{i} d x_{i}$ etc.

Without loss of generality, we assume that $\Pi\left(p_{1}\right)=\Pi\left(p_{2}\right)=0 \in \mathbb{R}^{n}$. By the assumption, there exists a diffeomorphism germ $\Phi:\left(\mathbb{R}^{n} \times \mathbb{R}, 0\right) \longrightarrow\left(\mathbb{R}^{n} \times \mathbb{R}, 0\right)$ of the form $\Phi(x, t)=\left(\phi_{1}(x), t+\alpha(x)\right)$ such that $\widehat{\Phi}\left(\mathscr{L}_{1}\right)=\mathscr{L}_{2}$. Then we have $\Phi^{-1}(x, t)=$ $\left(\phi_{1}^{-1}(x), t-\alpha\left(\phi_{1}^{-1}(x)\right)\right)$, so that the Jacobi matrix is

$$
J_{\Phi(x)} \Phi^{-1}=\left(\begin{array}{cc}
\frac{\partial \phi_{1}^{-1}}{\partial x}\left(\phi_{1}(x)\right) & 0 \\
-\frac{\partial \alpha \circ \phi_{1}^{-1}}{\partial x}\left(\phi_{1}(x)\right) & 1
\end{array}\right) .
$$

It follows that

$$
\widehat{\Phi}((x, t),[\xi: \tau])=\left(\Phi(x, t),\left[\xi \cdot \frac{\partial \phi_{1}^{-1}}{\partial x}\left(\phi_{1}(x)\right)-\tau \frac{\partial \alpha \circ \phi_{1}^{-1}}{\partial x}\left(\phi_{1}(x)\right): \tau\right]\right) .
$$

Since $\tau \neq 0$,

$$
\left[\xi \cdot \frac{\partial \phi_{1}^{-1}}{\partial x}\left(\phi_{1}(x)\right)-\frac{\partial \alpha \circ \phi_{1}^{-1}}{\partial x}\left(\phi_{1}(x)\right): \tau\right]=\left[-\frac{\xi}{\tau} \cdot \frac{\partial \phi_{1}^{-1}}{\partial x}\left(\phi_{1}(x)\right)+\frac{\partial \alpha \circ \phi_{1}^{-1}}{\partial x}\left(\phi_{1}(x)\right):-1\right] \text {. }
$$

We consider the graph-like affine coordinates $((x, t), p) \in J_{G A}^{1}\left(\mathbb{R}^{n}, \mathbb{R}\right)$, where $p=-\frac{\xi}{\tau}$. Then we have $\widehat{\Phi}\left(J_{G A}^{1}\left(\mathbb{R}^{n}, \mathbb{R}\right)\right)=J_{G A}^{1}\left(\mathbb{R}^{n}, \mathbb{R}\right)$ and

$$
\widehat{\Phi}((x, t), p)=\left(\phi_{1}(x), t+\alpha(x), p \cdot \frac{\partial \phi_{1}^{-1}}{\partial x}\left(\phi_{1}(x)\right)+\frac{\partial \alpha \circ \phi_{1}^{-1}}{\partial x}\left(\phi_{1}(x)\right)\right) .
$$

We now define a map $\widetilde{\phi_{1}}: T^{*} \mathbb{R}^{n} \longrightarrow T^{*} \mathbb{R}^{n}$ by

$$
\widetilde{\phi}_{1}(x, p)=\left(\phi_{1}(x), p \cdot \frac{\partial \phi_{1}^{-1}}{\partial x}\left(\phi_{1}(x)\right)+\frac{\partial \alpha \circ \phi_{1}^{-1}}{\partial x}\left(\phi_{1}(x)\right)\right) .
$$

Since $\widehat{\Phi}$ is a contact diffeomorphism germ, there exists a function germ $\mu: J_{G A}^{1}\left(\mathbb{R}^{n}, \mathbb{R}\right) \longrightarrow$ $\mathbb{R}$ with $\mu(x, t, p) \neq 0$ such that $\widehat{\Phi}^{*} \theta=\mu \theta$. Therefore, we have

$$
d t+d \alpha-{\widetilde{\phi_{1}}}^{*}(p \cdot d x)=\mu(d t-p \cdot d x)=\mu d t-\mu(p \cdot d x),
$$

so that $\mu \equiv 1$. It follows that $-p \cdot d x=d \alpha-\widetilde{\phi}_{1}{ }^{*}(p \cdot d x)$. Thus we have

$$
\widetilde{\phi}_{1}^{*}(\omega)={\widetilde{\phi_{1}}}^{*}(d(p \cdot d x))=d \widetilde{\phi}_{1}^{*}(p \cdot d x)=d(p \cdot d x)=\omega .
$$

This means that $\widetilde{\phi_{1}}$ is a symplectic diffeomorphism germ (i.e., Lagrangian diffeomorphism germ). Since $\Pi \circ \widehat{\Phi}\left|J_{G A}^{1}\left(\mathbb{R}^{n}, \mathbb{R}\right)=\widetilde{\phi}_{1} \circ \Pi\right| J_{G A}^{1}\left(\mathbb{R}^{n}, \mathbb{R}\right)$, we have $\Pi\left(\mathscr{L}_{2}\right)=\Pi \circ \widehat{\Phi}\left(\mathscr{L}_{1}\right)=$ $\widetilde{\phi}_{1}\left(\Pi\left(\mathscr{L}_{1}\right)\right)$.

By Propositions 4.1 and 4.2, we conclude the following theorem. 
Theorem 4.3 The graph-like Legendrian unfoldings $\left(\mathscr{L}_{1}, p_{1}\right)$ and $\left(\mathscr{L}_{2}, p_{2}\right)$ are $S . P^{+}$Legendrian equivalent if and only if the Lagrangian submanifold germs $\left(\Pi\left(\mathscr{L}_{1}\right), \Pi\left(p_{1}\right)\right)$ and $\left(\Pi\left(\mathscr{L}_{2}\right), \Pi\left(p_{2}\right)\right)$ are Lagrangian equivalent.

As a corollary of the above theorem, we have the following result:

Corollary 4.4 ([9]) The graph-like Legendrian unfolding $(\mathscr{L}, p)$ is S.P ${ }^{+}$-Legendrian stable if and only if the Lagrangian submanifold germ $(\Pi(\mathscr{L}), \Pi(p))$ is Lagrangian stable.

In [9] we apply the infinitesimal characterization of $S . P^{+}$-Legendrian stability. However, the assertion of Corollary 4.4 follows directly from Theorem 4.3.

On the other hand, by Corollary 4.4 and the fact $\pi \circ \Pi=\pi_{1} \circ \bar{\pi}$, the set of Legendrian singular points of a graph-like Legendrian unfolding $\mathscr{L}$ coincides with the set of Lagrangian singular points of $\pi \mid \Pi(\mathscr{L})$. Moreover, for generic graph-like Legendrian unfolding germ $(\mathscr{L}, p)$, the set of singular points of $\bar{\pi} \mid \mathscr{L}$ is nowhere dense. Hence we can apply Proposition 3.1 to our situation and obtain the following geometric interpretation of Lagrangian equivalence as a corollary of Theorem 4.3.

Theorem 4.5 Let $\left(\mathscr{L}_{1}, p_{1}\right)$ and $\left(\mathscr{L}_{2}, p_{2}\right)$ be graph-like Legendrian unfolding germs such that the sets of singular points of $\bar{\pi}\left|\mathscr{L}_{1}, \bar{\pi}\right| \mathscr{L}_{2}$ are nowhere dense respectively. Then the following conditions are equivalent:

(1) $\left(\Pi\left(\mathscr{L}_{1}\right), \Pi\left(p_{1}\right)\right)$ and $\left(\Pi\left(\mathscr{L}_{2}\right), \Pi\left(p_{2}\right)\right.$ are Lagrangian equivalent.

(2) Graph-like wave fronts $\left(W\left(\mathscr{L}_{1}\right), \bar{\pi}\left(p_{1}\right)\right)$ and $\left(W\left(\mathscr{L}_{2}\right), \bar{\pi}\left(p_{2}\right)\right)$ are S.P $^{+}$-diffeomorphic.

We remark that the condition (1) implies the contain (2) in the above theorem without any assumptions.

Let $(\mathscr{L}, p)$ be a graph-like Legendrian unfolding germ. We consider a representative $\widetilde{\mathscr{L}}$ of $(\mathscr{L}, p)$ on $\bar{\pi}^{-1}(W)$, where $W \subset \mathbb{R}^{n} \times \mathbb{R}$ is an open neighborhood of $\bar{\pi}(p) \in \mathbb{R}^{n} \times \mathbb{R}$. Then we have a representative $W(\widetilde{\mathscr{L}})=W(\widetilde{\mathscr{L}}) \cap W$ of the set germ $(W(\mathscr{L}), \bar{\pi}(p))$.

\section{References}

[1] V. I. Arnol'd, S. M. Gusein-Zade and A. N. Varchenko, Singularities of Differentiable Maps vol. I. Birkhäuser, Basel 1986.

[2] V. I. Arnol'd, Singularities of Caustics and Wave Fronts. Kluer Academic Publishers, Dordrecht 1990.

[3] J. J. Duistermaat, Oscillatory Integrals, Lagrange Immersions and Unfolding of Singularities. Communications of Pure and Applied Math. XXVII (1974), 207-281.

[4] L. Hörmander, Fourier Integral Operators,I. Acta. Math. 128 (1972), 79-183.

[5] S. Izumiya, Perestroikas of optical wave fronts and graphlike Legendrian unfoldings. J. Differential Geom. 38 (1993), 485-500.

[6] S. Izumiya, Completely integrable holonomic systems of first-order differential equations. Proc. Royal Soc. Edinburgh 125A (1995), 567-586. 
[7] S. Izumiya and M. Takahashi, Spacelike parallels and evolutes in Minkowski pseudospheres. Journal of Geometry and Physics. 57 (2007), 1569-1600.

[8] S. Izumiya and M. Takahashi, Caustics and wave front propagations: Applications to differential geometry. Banach Center Publications. Geometry and topology of caustics. 82 (2008) 125-142.

[9] S. Izumiya and M. Takahashi, Pedal foliations and Gauss maps of hypersurfaces in Euclidean space. Journal of Singularities. 6 (2012) 84-97.

[10] J. F. Nye, Natural focusing and fine structure of light. Insitute of Physics Publishing, Bristol and Philadelphia, 1999

[11] V. M. Zakalyukin, Reconstructions of fronts and caustics depending one parameter and versality of mappings, J. Sov. Math. 27 (1984), 2713-2735.

[12] V.M. Zakalyukin, Envelope of Families of Wave Fronts and Control Theory. Proc. Steklov Inst. Math. 209 (1995), 114-123.

\section{SHYUiCHI IZUMiYa}

Department of MATHEMATICS

HOKKAIDO UNIVERSITY

SAPPORO 060-0810, JAPAN

e-mail: izumiya@math.sci.hokudai.ac.jp 